Revue internationale de l'économie sociale

Recma

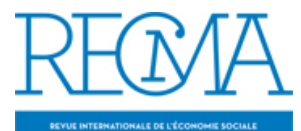

\title{
Après la faillite de Co-op Bank, quelles perspectives pour la gouvernance des coopératives bancaires?
}

Jean-Louis Bancel et Olivier Boned

Numéro 333, juillet 2014

URI : https://id.erudit.org/iderudit/1026047ar

DOI : https://doi.org/10.7202/1026047ar

Aller au sommaire du numéro

Éditeur(s)

Association Recma

ISSN

1626-1682 (imprimé)

2261-2599 (numérique)

Découvrir la revue

Citer cet article

Bancel, J.-L. \& Boned, O. (2014). Après la faillite de Co-op Bank, quelles perspectives pour la gouvernance des coopératives bancaires ? Revue internationale de l'économie sociale, (333), 108-113.

https://doi.org/10.7202/1026047ar 


\title{
APRĖS LA FAILLITE DE CO-OP BANK, QUELLES PERSPECTIVES POUR LA GOUVERNANCE DES COOPÉRATIVES BANCAIRES?
} par Jean-Louis Bancel* et Olivier Boned**

\author{
* Président de l'Association \\ internationale des banques \\ coopératives (AIBC). \\ ** Président du Centre des \\ jeunes, des dirigeants, des \\ acteurs de l'économie sociale \\ (CJDES).
}

Depuis la vague des démutualisations de l'époque thatchérienne (1997), la crise que traverse The Co-operative Bank est une des plus importantes que subisse le secteur européen des banques coopératives. The Co-operative Group, dont le siège est à Manchester (Royaume-Uni), comprend en son sein des activités diverses, que ce soit dans la pharmacie, l'agriculture, les pompes funèbres ou encore la banque au travers de The Co-operative Bank. Cette dernière, afin de changer de taille et d'acquérir un réseau de distribution plus dense, s'est engagée en 2009 dans le rachat du groupe immobilier Britannia. A l'audit qui aurait largement minimisé les risques, s'est ajouté un retournement du marché immobilier. La banque s'est ensuite engagée dans le rachat de 630 agences de Lloyds Banking Group, qu'elle ne parviendra pas à finaliser. Dans le même temps, la très forte augmentation des exigences totales de capital par le régulateur national, passant de 1,9 milliard de livres à 3,4 milliards, ainsi que les errements de son président ont été autant de facteurs qui ont entraîné la banque dans une spirale infernale. Cette plongée a nécessité une recapitalisation de 1,5 milliard de livres, puis de 400 millions. La banque est alors passée aux mains de ses créanciers. Un risque de contagion a émergé: l'ensemble du mouvement coopératif peut ainsi être contesté et fragilisé.

Ce qui arrive à cette banque anglaise sonne comme un appel à poursuivre la construction d'une activité économique en accord avec les attentes des membres coopérateurs. Le chief executive de Phone Co-op rappelait que, de la période des démutualisations, les coopératives survivantes sont ressorties revigorées. L'enjeu aujourd'hui est de renouer les liens entre les membres coopérateurs et leurs dirigeants, réaffirmer en somme le modèle coopératif de gouvernance, une position qui souligne l'importance de la dimension humaine et culturelle de la démocratie en entreprise. Celle-ci fonctionne si elle peut s'exercer dans un cadre local, de manière directe entre la structure et le membre. Cela n'est pas antinomique de l'existence de grandes entités coopératives ou mutualistes, pour autant que les membres 
aient une réelle capacité d'influence. La notion de proximité doit alors être réaffirmée, pour amener les dirigeants coopératifs à rester au contact de la réalité économique et des enjeux. Les difficultés de Co-op Bank mettent en évidence une tendance à évincer du cœur du système les administrateurs des banques. Les coopérateurs doivent prendre conscience que l'héritage et l'avenir de la coopération sont en jeu.

\section{L'OUBLI DE LA DIMENSION COOPÉRATIVE}

Durant les années 80 et 90, les banques coopératives avaient pour objectif d'être reconnues par les autorités et l'opinion publique comme des banques de pleine capacité, et non plus comme de simples établissements de crédit. Certaines se laisseront entraîner dans cette période d'intense activité économique, de développement de la finance et de course aux sièges sociaux de verre et de métal, symboles de réussite. Durant ces années, les banques coopératives " ont voulu faire mieux que les banques commerciales. D’où des courses indécentes à la baisse du coefficient d'exploitation, d'où une pression ailleurs inconnue à la vente ou encore une volonténä̈ve d'afficher du résultat, à n'importe quelle condition et, surtout, sans objet défini » ${ }^{(1)}$. La notion

(1) Juvin H., 2013, « L'avenir des banques mutualistes ou coopératives ", Banque et Stratégie, $\mathrm{n}^{\circ} 317$. de sociétaire fut délaissée au profit de celle plus moderne et prétendument plus professionnelle de « client ». La loi bancaire de 1984 a ouvert une brèche: il était désormais impossible d'imposer à des clients de devenir systématiquement sociétaires. Les dimensions économique, d'un côté, et démocratique, de l'autre, coexisteront, mais ne seront plus totalement unies. Le "mouvement » et l'" économique » sont, depuis, les deux jambes sur lesquelles avance la banque coopérative. Cet écart est devenu une faille grandissante où des représentants d'une minorité de sociétaires semblaient réduits à entériner les choix de « vrais » professionnels. Pour autant, la perspective de voir les avoirs (capital social et réserves impartageables) de la minorité des sociétaires venir renflouer les engagements pris par la technostructure (mécanisme dit du bail in imposé par les réformes bancaires pour éviter l'injection d'argent public) au profit d'une majorité de clients indifférents à la nature coopérative de la banque amène à s’interroger sur les responsabilités. Reste à clarifier qui incarne, dans une banque coopérative, la tête, donc la décision et la responsabilité de rendre des comptes aux sociétaires.

\section{LES VALEURS RETROUVÉES}

\section{La RSE relance la « coopérative attitude »}

Au début des années 2000, le sociétariat est en berne. Certaines banques coopératives présentent des taux de sociétariat aux alentours de $50 \%$ : un client sur deux n'est plus coopérateur. Or, les coopératives n'ont de raison d'être que par l'adhésion de leurs membres, le partage du projet et le vote démocratique, dont on prend conscience alors qu'ils sont menacés. Ce réveil provient de mouvements internes, mais également d'épiphénomènes externes. La notion de responsabilité sociale de l'entreprise (RSE), qui date de la première moitié du $\mathrm{XX}^{\mathrm{e}}$ siècle, est en forte progression auprès d'entreprises 
du monde entier. Les méthodes d'évaluation amènent les banques coopératives à réinterroger leurs pratiques. Les structures commencent à y percevoir une occasion de renouer avec leurs valeurs coopératives. La RSE apparaît alors comme un levier ou un révélateur de leur action positive sur leur environnement, au sens large, et sur leurs sociétaires-clients en particulier. Ce qui est alors mentionné dans les rapports, les brochures et les films mélange dimension coopérative, valeurs mutualistes et gouvernance démocratique. Dans cet élan de redécouverte, les banques coopératives commencent à affirmer que la RSE est inscrite dans leur ADN. Si cela doit être regardé avec prudence, cette dynamique va avoir le mérite de les pousser à réinterroger leur modèle et leurs valeurs. Elles s'engagent sur ce qui marque leur différence, leurs origines et leur raison d'être, à savoir être au service de leurs membres et de la société. Des communications visent à démontrer que l'épargne locale vient enrichir la communauté locale, que la banque du territoire développe le territoire, que l'argent gagné par les épargnants ne sert pas la spéculation financière. L'engouement est également européen: l'Association européenne des banques coopératives (EACB) se lance dans l'animation d'un groupe de travail sur la RSE avec de multiples banques. En moins d'une dizaine d'années, les banques coopératives s'approprient ce concept qui valorise leurs pratiques et en font une marque de différenciation. Etre une coopérative devient, dans les publicités télévisuelles, la garantie d'un service responsable au plus proche des gens. La proximité est à nouveau fondamentale dans un monde globalisé. Trois quarts des sièges sociaux des coopératives françaises sont basés hors région parisienne, au plus près donc des coopérateurs. La « coopérative attitude " prend son envol.

\section{Quelle spécificité pour les coopératives?}

Néanmoins, les contingences de gestion et de contrôle s'accroissent, notamment avec le double crash des années 2000: Enron, puis Lehman Brothers. Du premier épisode, sortiront les obligations de transparence des marchés, de contrôle des dirigeants, de mise en place de comités d'audit, de procédures de contrôle interne plus fortes. Le deuxième scandale entachera durablement la réputation du système bancaire. Les banques coopératives tireront cependant leur épingle du jeu et renforceront au passage leurs parts de marché et le nombre de leurs clients.

Or, leur équilibre et leur performance tiennent à cet élément fondamental de mieux en mieux abordé, au travers de rapports successifs - notamment ceux qui ont été présidés par Etienne Pflimlin, dans le cadre de l’Institut Français des administrateurs -, à savoir la gouvernance.

\section{DE LA NÉCESSITÉ DE RAPPELER LA GOUVERNANCE DÉMOCRATIQUE DES BANQUES COOPÉRATIVES}

\section{" Par et pour leurs membres "}

Les coopératives sont constituées par et pour leurs membres. Les notions de contrôle et de gestion de l'entreprise sont donc liées au fait que des personnes s'associent pour collectivement "réduire, au bénéfice de leurs membres et 
par l'effort commun de ceux-ci, le prix de revient et, le cas échéant, le prix de vente de certains produits ou de certains services, en assumant les fonctions des entrepreneurs ou des intermédiaires dont la rémunération grèverait ce prix de revient » (art. 1, al. 1, loi 1947). Seuls les membres peuvent bénéficier des services de leur coopérative, et ils doivent garder le contrôle sur elle. Ainsi, comme en dispose l'article 6 de la loi de 1947, "les coopératives sont administrées pardes mandataires nommés pour six ans au plus parl'assemblée générale des membres et révocables par elle».

Au niveau de la gouvernance de tête des banques coopératives françaises, l'élection des administrateurs par l'assemblée générale des sociétaires est une clé de voûte. Les administrateurs représentent les intérêts des sociétaires et rendent compte de leurs mandats lors des élections en assemblée générale. Le président est généralement élu par le conseil d’administration, parfois par l'assemblée générale (au Crédit mutuel, par exemple).

Il est évident que les présidents et les administrateurs élus ne peuvent pas s'occuper de la gestion au quotidien, qui est confiée à un directeur général, mais un élément fondamental est à souligner: si le directeur général assure la direction de la société (art. L512-49, Code monétaire et financier), il est désigné et donc contrôlé, évalué et révocable par le conseil et son président. Ainsi, l'exercice de son pouvoir se fait dans le cadre des orientations fixées par le conseil. Le président occupe une fonction de définition et de contrôle de la stratégie, d'où l'importance qu'il peut jouer dans le contrôle interne de l'organisation coopérative. Les organes centraux des banques coopératives sont chargés certes de la cohérence entre la stratégie et la solidité du groupe, mais aussi de la représentation, de la cohésion et du respect des règles au sein du réseau et du contrôle interne: ils "veillent à l'application des dispositions législatives et réglementaires propres à ces établissements et sociétés et exercent un contrôle administratif, technique et financier sur leur organisation et leur gestion » (art. L511-31, Code monétaire et financier). Les compétences sont ainsi réparties, voire personnifiées entre deux acteurs: le président et le directeur général, et l'équilibre de la gouvernance des banques coopératives repose sur ce tandem. Dans sa position du 20 juin 2014, l'Autorité de contrôle prudentiel et de résolution (ACPR), en confirmant que le président n'est pas un "dirigeant effectif ", marginalise le président du conseil d'administration et affaiblit la force du modèle coopératif. Même si elle souligne "l’importance cruciale du conseil d'administration et de son président pour assurer une gouvernance efficace et harmonieuse », ses préconisations vont, en substance, à l'encontre de cette déclaration qui veut donner l'impression d'avoir entendu les arguments du monde mutualiste et coopératif.

De plus, au-delà de la stratégie, le contrôle est de la responsabilité des élus. S'il est indispensable de veiller à ce que ces derniers occupent pleinement leurs fonctions, en démontrant les compétences nécessaires, il apparaît dangereux de déléguer à la direction générale la totalité du contrôle de l'organisation. Cela irait à l'encontre des textes réglementaires, mais aussi et surtout de la logique coopérative. Les élus seraient ainsi écartés de leur rôle et cela viderait totalement la raison d'être des banques coopératives au service de leurs membres et gouvernées par eux. 


\section{L'ACPR à contre-courant des coopérateurs}

Si un président de banque coopérative ne doit pas être un dirigeant au quotidien, autrement dit engagé dans la mise en œuvre au jour le jour des actions de la banque, il doit rester un dirigeant réel, effectif, engagé dans la définition de la stratégie et le contrôle de sa mise en œuvre au bénéfice des sociétaires. Cet équilibre est simple, pratiqué de longue date dans le mouvement coopératif et permet à ce dernier d'occuper la place qui est la sienne aujourd'hui. L’ACPR, en publiant une position le 29 janvier 2014, mentionne que «le cumul des fonctions de président du conseil d'administration et de directeur général n'est désormais plus possible» (2014-P-02). Pour justifier cela, elle s'appuie sur la directive européenne CRD4, entrée en vigueur au $1^{\mathrm{er}}$ janvier 2014, qui mentionne que «le président de l'organe de direction dans sa fonction de surveillance d'un établissement ne peut pas exercer simultanément la fonction de directeur général dans le même établissement, sauflorsqu'une telle situation est justifiée par l'établissement et approuvée par les autorités compétentes » (directive 2013-36-UE, art. 88, al.1).

Or, ce principe ne pose pas de problème de fonctionnement aux banques coopératives qui ont, depuis longtemps, dissocié la répartition des pouvoirs entre le président et le directeur général. Seulement, l'ACPR poursuit dans une deuxième position - également du 29 janvier $2014^{(2)}$-, visant à retirer au président sa qualité de dirigeant: "Les personnes qui assurent la direction effective d'un établissement, aussi désignées sous le terme de "dirigeants responsables", doivent disposer des pouvoirs les plus larges. Dans une société anonyme, outre le directeur général, qui dispose en effet [...] des pouvoirs les plus étendus pour agir en toute circonstance au nom de la société(art. L225-56 du Code de commerce), le deuxième dirigeant responsable doit en principe être un directeur

(2) Cette décision a été remplacée sans modification significative sur le fond par une position 2014-P-07 du 20 juin 2014, à la suite d'un recours gracieux des banques coopératives. général délégué» (2014-P-03). Cette vision des choses pose deux problèmes majeurs. Tout d'abord, elle vient déstabiliser en profondeur le fonctionnement équilibré des banques coopératives. Ensuite, son interprétation peut être largement soumise à débat, juridique, mais aussi sur les présupposés sociologiques qu'elle emporte concernant la caractérisation des élus coopératifs. La directive elle-même, dans son considérant 53 , reconnaît que l'un des objectifs poursuivis en matière de gouvernance est un véritable équilibre des pouvoirs, ce à quoi les banques coopératives s'emploient dans la dissociation des fonctions, la règle du double regard entre le président et le directeur général et la répartition des rôles. La directive européenne laisse dans son considérant 55 une liberté d'organisation interne qui ne remet en aucun cas en cause le positionnement existant du président dans les banques coopératives: «Différentes structures de gouvernance sont utilisées au sein des Etats membres. Dans la plupart des cas, une structure unitaire ou duale est utilisée. Les définitions utilisées dans la présente directive visent à prendre en compte l'ensemble des structures existantes, sans privilégier l'une d'entre elles en particulier. Elles sont purement fonctionnelles et ont pour objet de fixer les règles en vue de parvenir à un certain résultat indépendamment du droit national des sociétés applicable à un établissement dans chaque Etat membre. Par conséquent, les définitions n’influent pas sur la répartition globale des compétences, conformément au droit national des sociétés » (directive 2013-36-UE). 
Cette expression persistante de l'autorité de supervision bancaire française ne manque pas de faire apparaître une césure politique entre elle et les millions de sociétaires des banques coopératives dont elle semble vouloir faire le bien malgré eux. La volonté de résistance exprimée par le recours gracieux déposé par les banques coopératives doit conduire celles-ci à mieux redéfinir le rôle et les missions du président, en d'autres termes renforcer sa position de stratège et de superviseur. La question de la compétence, au travers de la formation et du temps d'engagement dans l'organisation, doit également être un sujet traité en profondeur et en commun. Le scandale de The Co-operative Bank, en Angleterre, ne peut que nous y inviter.

\section{CONCLUSION}

De la crise est née la prise de conscience de la nécessité de se démarquer, de revenir sur la base fondamentale des banques coopératives, c'est-à-dire le sociétaire: "Considérécomme un handicap durant les années de financiarisation, le "sociétariat" est de retour dans les banques mutualistes et coopératives depuis que la crise financière a minéla confiance dans le système bancaire et en particulier dans la gouvernance des établissements " ${ }^{(3)}$. Cette prise

(3) Gomez P.-Y., « Les banques coopératives jouent-elles vraiment leur rôle? ", Le Monde, 18 juillet 2013. de conscience divise alors et fait débat. Certains voient dans cette nouvelle attitude une démarche marketing, commerciale, visant à se distinguer des concurrents, voire à regagner en pureté. D’autres, comme les banques coopératives elles-mêmes, redécouvrent une dimension qu'elles avaient égarée et qui leur permet de reconstruire une confiance altérée par la crise et les investissements spéculatifs, bien éloignés des préoccupations des sociétaires. La reconquête ne pourra passer que par la restauration du lien avec les sociétaires et le retour aux positions de proximité, qui étaient la base de la légitimité des banques coopératives et des coopératives dans leur ensemble, au plus proche des sociétaires, de leurs besoins, de leurs attentes et de leur vote. Ces organisations retrouvent ou redécouvrent une part de leur identité, qui est également réappropriée par les individus. Une banque qui appartient à ses clients laisse l'opportunité d'en devenir acteur. Même si les normes et la professionnalisation des métiers en appellent à plus d'implication de salariés professionnels, ces organisations laissent l'opportunité pour des individus de se réinvestir dans des projets collectifs. Il est étonnant, d'ailleurs, que les banques coopératives ne soient pas associées à ce qu'il est désormais courant de qualifier de « révolution collaborative ", à travers le crowdfunding, l'appel direct au public ou encore Wikipédia, alors que cette forme de coopération correspond largement à l'identité coopérative ${ }^{(4)}$.

(4) Juvin H., 2013, op. cit. Si un élément est à retenir dans l'économie sociale, c'est bien la gestion collective d'un projet, ce qui nous amène à souligner combien le retour de la démocratie dans l'économie peut avoir d'avantages. Si elle n'est pas parfaite, elle demeure le mécanisme de protection des intérêts de chacun et de l'expression de tous. 